\title{
REMOÇÃO DE MATÉRIA ORGÂNICA E NUTRIENTES DE ESGOTO DOMÉSTICO POR WETLAND HORIZONTAL DE FLUXO SUBSUPERFICIAL NA ESTAÇÃO DE TRATAMENTO DE APARECIDA - CAMPOS NOVOS, SC
}

\author{
Lucas Silva Lourenço ${ }^{1}$, Eduardo Bello Rodrigues ${ }^{1}$, Marcelo Alves Moreira ${ }^{1}$, Everton Skoronski ${ }^{1}$
}

\begin{abstract}
RESUMO - A falta de sistemas de tratamento para o esgoto doméstico adequados às condições dos pequenos municípios brasileiros ainda é uma realidade. Diante disso, este foi um trabalho exploratório de seis meses (agosto de 2016 a janeiro de 2017) que visou determinar a eficiência de uma estação de tratamento de esgoto (ETE) no município de Campos Novos (SC) do tipo wetlands construídos. O sistema utilizado foi uma unidade de fluxo horizontal subsuperficial operando como pós-tratamento de esgoto doméstico em filtro anaeróbio. Foi utilizado Typha sp. (Taboa) como espécie de planta para absorção dos nutrientes. A eficiência do referido sistema em termos de remoção de matéria orgânica e de sólidos mostrou-se estável, com eficiências médias de 62, 64 e 50\% para DQO, DBO e SST, respectivamente. O sistema apresentou uma redução em 2 unidades logarítmicas para coliformes totais, com eficiência de remoção de 69,7\%. A remoção de nutrientes, após início promissor, mostrou-se instável devido a colmatação, com remoção média de 31,8 e 22,4\% para $\mathrm{N}_{\text {Total }}$ e $\mathrm{P}_{\text {Total }}$, respectivamente. Durante o período monitorado a ETE atendeu parcialmente as legislações vigentes (Resolução CONAMA n 430/2011 e Lei estadual n 14675/2009 (FATMA)) quanto ao descarte de efluente doméstico em corpo hídrico. A exceção foi DBO e $\mathrm{P}_{\text {Total }}$ que estavam acima dos padrões estabelecidos pela FATMA nos dois últimos meses, contudo dentro dos limites estabelecidos pela CONAMA. Apesar da precoce manutenção corretiva e preventiva devido a colmatação, a utilização de wetlands construídos de escoamento horizontal de fluxo subsuperficial é uma alternativa para o tratamento de esgoto doméstico.
\end{abstract}

Palavras chave: fluxo horizontal, tratamento de esgoto doméstico, Typha sp., wetland construído.

\section{REMOVAL OF ORGANIC MATTER AND NUTRIENTS FROM DOMESTIC SEWAGE BY CONSTRUCTED WETLANDS IN APARECIDA TREATMENT STATION - CAMPOS NOVOS, SC, BRAZIL}

\begin{abstract}
The lack of treatment systems for domestic sewage adequate to the conditions of small Brazilian municipalities is still a reality. Therefore, this is an exploratory work of 6 months (August 2016 to January 2017) that aimed to determine the efficiency of a sewage treatment plant in the municipality of Campos Novos, SC, constructed wetlands (CWS) type. The system used was a horizontal subsurface flow unit operating with domestic sewage post-treatment with the anaerobic filter. Typha sp. (Taboa) was used as a plant species for nutrient absorption. The efficiency of this system in terms of removal of organic matter and suspended solids was stable, with efficiencies of 62, 64 and 50\% for COD, BOD and SST, respectively. The nutrients uptake by taboa was unstable in the last months, after a promising onset, with the removal of 31.8 and $22.4 \%$ for the total $N$ and total $P$, respectively. The treatment system reduced 2 log units to the total coliforms, with $69.7 \%$ of efficiency. The CWS from Aparecida complied with the current legislation (CONAMA Resolution No. 430/2011 and State Law No. 14675/2009 (FATMA)) regarding the disposal of domestic effluent in the waterbody. However, in the last two months of monitoring the BOD and total $P$ did not comply with the standards established by FATMA. Nevertheless, they were lower than the limits established by CONAMA. Despite the early corrective and preventive maintenance to reduce clogging, the use of horizontal subsurface flow constructed wetlands is a feasible alternative to promote the treatment of domestic sewage.
\end{abstract}

Keywords: constructed wetlands, horizontal flow, Thypha sp., treatment of domestic sewage.

\footnotetext{
${ }^{1}$ Universidade do Estado de Santa Catarina.E-mail: lucas22louren@hotmail.com; eduardo.rodrigues@udesc.br; marcelo.moreira@udesc.br; everton.skoronski@udesc.br
} 


\section{INTRODUÇÃO}

O saneamento básico é um conjunto de serviços, infraestrutura e instalações operacionais de abastecimento de água, esgotamento sanitário, limpeza urbana, drenagem urbana, manejos de resíduos sólidos e de águas pluviais que tem por objetivo prevenir doenças, promover a saúde e melhorar a qualidade de vida da população (BRASIL, 2007). No Brasil, a implantação do saneamento básico ainda apresenta muitas dificuldades, entre elas, destacam-se a coleta e o tratamento de efluentes. Nesse sentido, estimase que o Brasil tenha 207,6 milhões de habitantes distribuídos em 5.570 municípios e apenas 50,3\% da população tem acesso à coleta de esgoto, ou seja, mais de 100 milhões de brasileiros não tem a acesso a este serviço e apenas $42,7 \%$ do esgoto coletado no país é tratado (SNIS, 2015; IBGE, 2017).

Os números apresentados pelo Sistema Nacional de Informações sobre Saneamento revelam que, 19,4\% dos municípios catarinenses dispõem de rede coletora de esgotos, porém, apenas $24,3 \%$ do esgoto coletado é tratado. Esses baixos índices podem ser respondidos pelo fato que, $77,6 \%$ das cidades catarinenses possuem uma população de até 20 mil habitantes, ou seja, são cidades pequenas, que possuem recursos limitados para um investimento e manutenção das redes e estação de tratamento de esgoto (ETE). Ao analisar o Brasil como um todo, percebe-se que a realidade é a mesma, ou seja, 3.809 municípios brasileiros $(68,4 \%)$ possuem uma população de até 20 mil habitantes, sendo necessário buscar soluções em que essas cidades possam implantar e manter um tratamento de esgoto adequado (SNIS, 2015; IBGE, 2017). Para isso, existem tecnologias eficientes e de baixo custo, ideais para essas pequenas cidades.

Um exemplo disso foi realizado na cidade de Campos Novos em Santa Catarina, que através da autarquia responsável pelo tratamento de esgotos do município, planejou efetivar o seu atendimento, estabelecendo, a partir da Lei municipal No 3.941/2013, normas na área de tratamento de efluentes para a implantação de novos loteamentos no município, a fim de buscar a universalização do acesso à rede coletora e ao tratamento. Assim, exige-se como última etapa de tratamento do esgoto a tecnologia wetlands construídos horizontais de fluxo subsuperficial (WHFSS), para todos os novos loteamentos urbanos que não são atendidos pelo serviço de coleta e tratamento de esgotos (CAMPOS NOVOS, 2013).
O sistema wetland construído (WC) é uma tecnologia empregada no tratamento de esgoto doméstico, através da utilização de plantas, microrganismos e material filtrante em que ocorrem processos físicos, químicos e biológicos (ANSARI et al., 2016). Estes incluem sedimentação, precipitação, adsorção às partículas do material filtrante, assimilação pelos tecidos das plantas e transformações microbiológicas (PHILIPPI \& SEZERINO, 2004). Esses sistemas apresentam por características a sua construção, operação e manutenção simplificadas (KIVAISI, 2001). Diante disso, os wetlands construídos têm um grande potencial para aplicação em pequenas comunidades, ideal para a maioria dos municípios brasileiros.

Diante do exposto estabeleceu-se como principal objetivo deste trabalho avaliar a série de sólidos dissolvidos suspensos (SST, SSV e SSF), nitrogênio $\left(\mathrm{N}_{\text {Total }}\right)$ e fósforo total $\left(\mathrm{P}_{\text {Total }}\right)$, demandas bioquímica (DBO) e química (DQO) de oxigênio e coliformes totais (CT) em um sistema de tratamento de esgoto doméstico do tipo wetland construído no município de Campos Novos/SC.

\section{MATERIALE MÉTODOS}

O sistema wetland foi implantado pelo Serviço Autônomo Municipal de Água e Esgoto (SAMAE) no bairro Aparecida do município de Campos Novos - SC com uma capacidade de tratamento equivalente de 3.400 pessoas. Esse sistema é de fluxo horizontal, com escoamento subsuperficial dividido em quatro unidades, que operam em paralelo, recebendo as mesmas vazões.

A Figura 1 apresenta as características físicas de construção de cada unidade de wetland construído (WC1, WC2, WC3 e WC4) e têm as seguintes dimensões: 13 metros de largura, 26 metros de comprimento e 0,7 $\mathrm{m}$ de profundidade, perfazendo uma área total de 338 $\mathrm{m}^{2}$ para cada sistema. Para o preenchimento do leito filtrante das unidades de wetland construído foi usado como padrão três diferentes granulometrias de material filtrante: brita $\mathrm{n}^{\circ} 1$, pedrisco e areia grossa. A pedra lascão, por apresentar uma granulometria maior, facilita a distribuição do esgoto dentro dos primeiros metros das unidades de WC. A macrófita utilizada foi a Thypha $s p$. , popularmente conhecida como Taboa. Este sistema entrou em operação em janeiro de 2016.

O esgoto doméstico referente ao bairro Aparecida é direcionado até a ETE, este por sua vez é submetido 


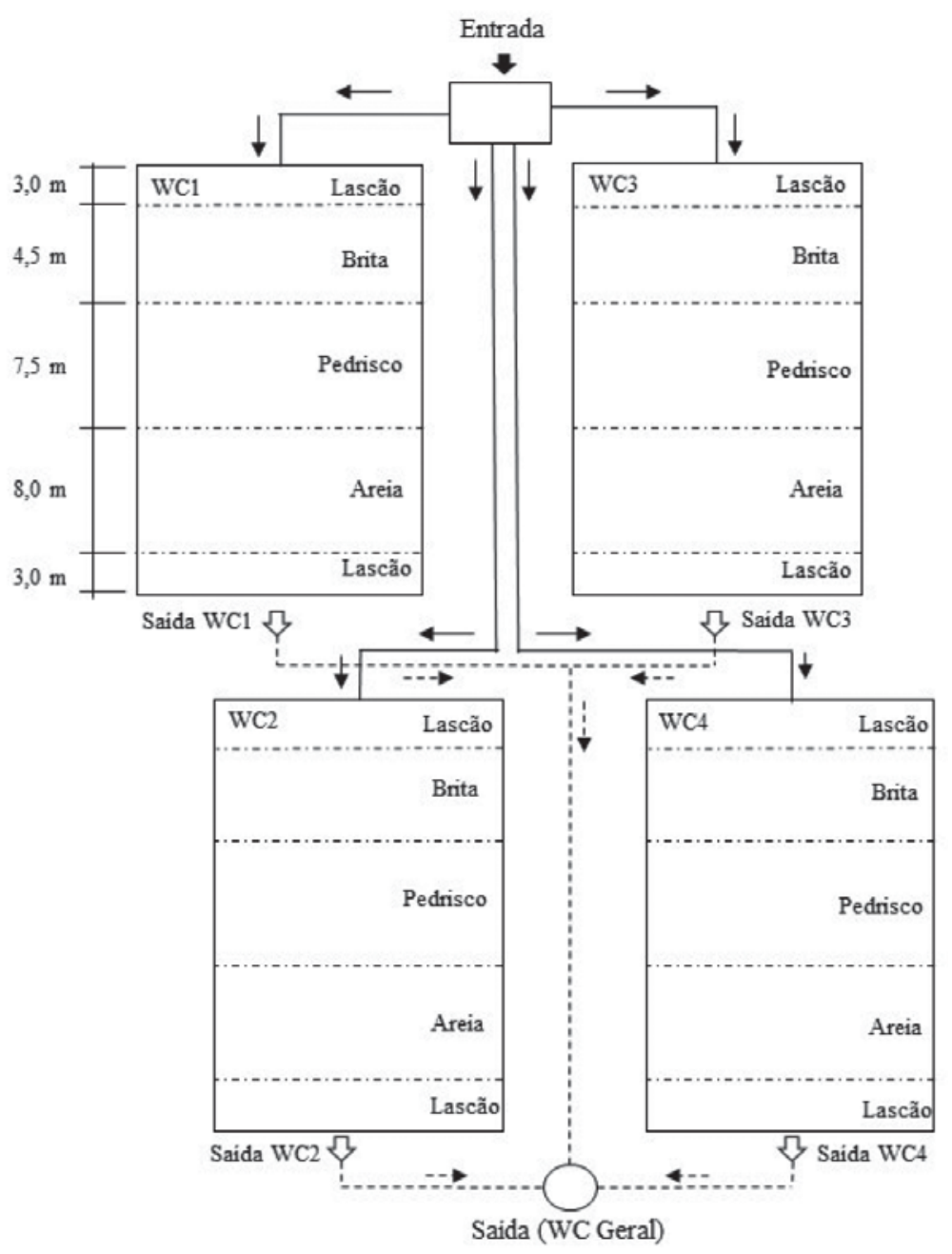

Figura 1 - Vista geral das quatro unidades de wetlands construídos de fluxo subsuperficial horizontal.

a três etapas de tratamento, que englobam, tratamento preliminar (gradeamento), reator de filtro anaeróbio e o wetland construído. Vale ressaltar que todo o sistema de tratamento de esgoto não consome energia, pois todo o esgoto é conduzido somente pela gravidade. Para a realização do monitoramento da estação, especificamente dos wetlands construídos foram feitas coletas mensais e amostragem única de esgoto em um ponto central de entrada e saída. Especificamente em duas unidades (WC1 e WC2) foram realizadas amostragens individuais, para avaliar a eficiência de cada uma. O período de monitoramento compreendeu seis meses, e este foi iniciado logo após a primeira poda da Taboa.
As análises dos parâmetros de qualidade do afluente e efluente das unidades de WC foram realizadas no Laboratório de Tratamento de Água e Resíduo - Labtrat do Departamento de Engenharia Sanitária e Ambiental do CAV/UDESC. Os seguintes parâmetros físico-químicos e microbiológicos foram avaliados, série de sólidos dissolvidos suspensos (SST, SSV e SSF), nitrogênio $\left(\mathrm{N}_{\text {Total }}\right)$ e fósforo total ( $\left.\mathrm{P}_{\text {Total }}\right)$, demandas bioquímica (DBO) e química (DQO) de oxigênio e coliformes totais (CT). As análises dos parâmetros citados foram realizadas de acordo com os procedimentos constantes no manual de Métodos laboratoriais de análises físico-químicas e microbiológicas - $4^{\mathrm{a}}$ edição, (Macedo, 2013) e Standard Methods for the Examination of Water and Wastewater 
(APHA, 1998) e os parâmetros foram comparados com as legislações vigentes (Resolução CONAMA n ${ }^{\circ} 430 /$ 2011 e Lei estadual do estado de Santa Catarina ${ }^{\circ}$ 14675/2009) (BRASIL, 2005; SANTACATARINA, 2009).

\section{RESULTADOS E DISCUSSÃO}

\section{Matéria orgânica}

A Figura 2 apresenta as séries temporais das concentrações efluentes de demanda química (DQO) e bioquímica(DBO) de oxigênio das seis campanhas realizadas nos meses de agosto de 2016 a janeiro de 2017.

Os wetlands construídos proporcionaram no período monitorado uma eficiência média de remoção de DQO de 49, 61 e 62\% para WC1, WC2 e WC geral, respectivamente e de DBO de 61, 71 e 64\% para WC1, WC2 e WC geral, respectivamente.

Na Figura 3 encontram-se os resultados (expressos em gráficos boxplot) do monitoramento de DBO e DQO dos efluentes das unidades de tratamento do filtro anaeróbio (entrada dos WC), WC1, WC2 e do WC geral (média das 4 unidades de WC).

Os gráficos boxplot mostram a representação gráfica de alguns dados estatísticos com valores mínimos, máximos e média, e quartis inferior (25\% percentis) e superior (75\% percentis). Em relação à concentração de DQO nos efluentes dos wetlands WC1, WC2 e WC geral, os valores obtidos nas amostras resultaram em $75 \%$ das amostras inferiores a $220 \mathrm{mg} \mathrm{L}^{-1}, 220,1 \mathrm{mg}$ $\mathrm{L}^{-1}$ e $256 \mathrm{mg} \mathrm{L}^{-1}, 25 \%$ das amostras superiores a 135 $\mathrm{mg} \mathrm{L}^{-1}, 135 \mathrm{mg} \mathrm{L}^{-1}$ e 97,2 $\mathrm{mg} \mathrm{L}^{-1}$ e $50 \%$ das amostras em 175 mg L $^{-1}$, 175 mg L $^{-1}$ e 135,1 mg L $^{-1}$ respectivamente.

Para as concentrações de DBO nos efluentes dos wetlands WC1, WC2 e WC geral, os valores obtidos nas amostras resultaram em 75\% das amostras inferiores a 67,5 $\mathrm{mg} \mathrm{L}^{-1}, 67,5 \mathrm{mg} \mathrm{L}^{-1}$ e $89 \mathrm{mg} \mathrm{L}^{-1}, 25 \%$ das amostras foram superiores a $10 \mathrm{mg} \mathrm{L}^{-1}, 10 \mathrm{mg} \mathrm{L}^{-1}$ e $20 \mathrm{mg} \mathrm{L}^{-1}$ e $50 \%$ das amostras em $42 \mathrm{mgL}^{-1}, 42 \mathrm{mgL}^{-1} \mathrm{e} 80 \mathrm{mg} \mathrm{L}^{-1}$, respectivamente.
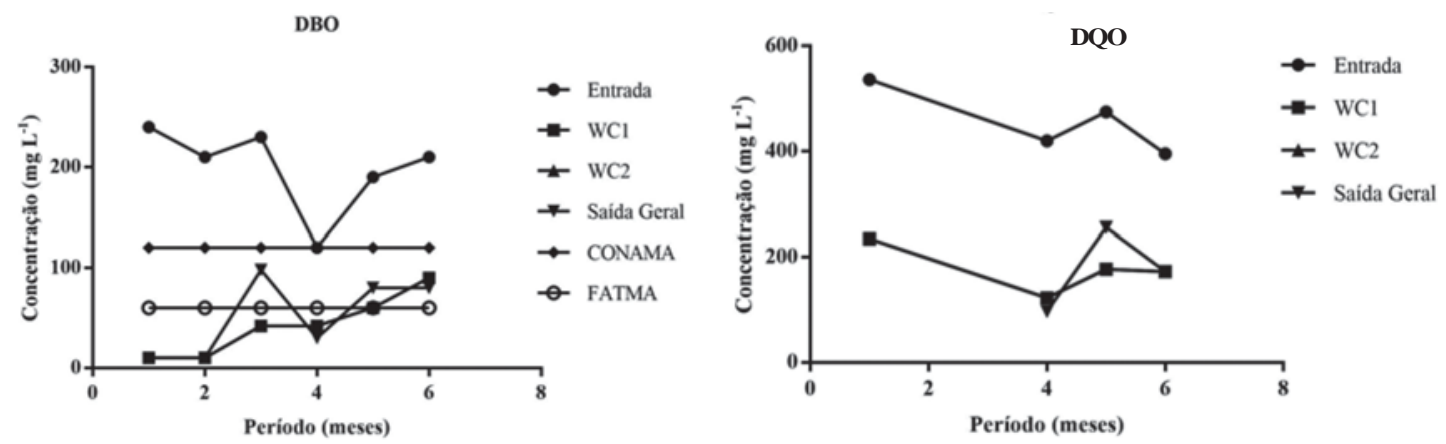

Figura 2 - Séries temporais das concentrações efluentes de DQO e DBO e comparação com a Lei do Estado de SC (FATMA) e a Resolução CONAMA.
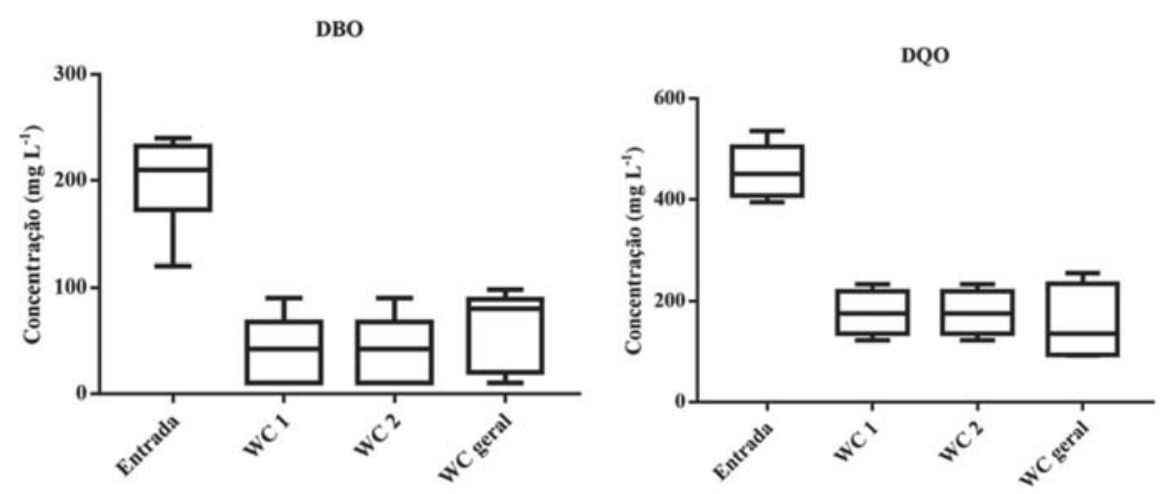

Figura 3 - Gráfico tipo boxplot, destacando as concentrações efluentes de DQO e DBO do filtro anaeróbio e dos wetlands construídos. 
A Resolução 430/2011 do Conselho Nacional de Meio Ambiente-CONAMA(BRASIL, 2011), estabelece a concentração de DBO de $120 \mathrm{mg} \mathrm{L}^{-1}$ ou $60 \%$ de eficiência de remoção, e a Lei estadual do Estado de Santa Catarina $n^{\circ}$ 14675/2009-FATMA(SANTA CATARINA, 2009) estabelece a concentração de $60 \mathrm{mg} \mathrm{L}^{-1}$ ou $80 \%$ de eficiência, o que foi atendido até o quarto mês de monitoramento. Após este período os WC passaram a lançar $80 \mathrm{mg} \mathrm{L}^{-1}$ de DBO, deixando de atender os padrões estabelecidos pela FATMA, mas ainda dentro dos limites estabelecidos pelo CONAMA.

O estudo de Colares e Sandri (2013) demonstraram eficiência de remoção similar de DBO para WC horizontais preenchido com filtro de brita e Taboa no tratamento de esgoto doméstico, sendo de 58\% de eficiência. Em outro estudo Konrad et al. (2015) também em WC horizontais obtiveram remoção média para DQO de 78\% para efluentes de esgoto doméstico. Já para Souza (2003) ao avaliar um wetland construído de fluxo horizontal subsuperficial com meio suporte de brita e vegetado com Taboa, obteve 38,4\% de remoção média de DQO em efluente de esgoto sanitário.

\section{Sólidos suspensos totais}

A Figura 4 apresenta a série temporal das concentrações efluentes de sólidos suspensos totais (SST), sólidos suspensos voláteis (SSV) e sólidos suspensos fixos (SSF).

Da mesma forma que para os parâmetros relativos à remoção de matéria orgânica, as concentrações de sólidos suspensos (SST, SSV e SSF) presentes no efluente do filtro anaeróbio e após o tratamento via wetlands construídos foram reduzidos. O efluente, após o filtro anaeróbio, apresentava concentrações médias de 578, 444 e $134 \mathrm{mg} \mathrm{L}^{-1}$, para SST, SSV e SSF respectivamente. Após os WC, o efluente geral da ETE de Aparecida apresentou concentrações médias de SST de $289 \mathrm{mg} \mathrm{L}^{-1}$, SSV de $240 \mathrm{mg} \mathrm{L}^{-1}$ e de SSF de $70 \mathrm{mg}$ $\mathrm{L}^{-1}$, apresentando uma eficiência média de remoção de $50 \%$ para SST, $46 \%$ para SSV e $48 \%$ para SSF.

A elevada concentração dos sólidos afeta diretamente o desempenho dos wetlands construídos, a longo prazo, não só na remoção dos sólidos, mas também para a maioria dos outros parâmetros, como por exemplo o nitrogênio. $\mathrm{O}$ aporte excessivo de sólidos nesses sistemas pode contribuir consideravelmente para a colmatação do meio filtrante e afetar o desempenho geral (De Paoli \& Von

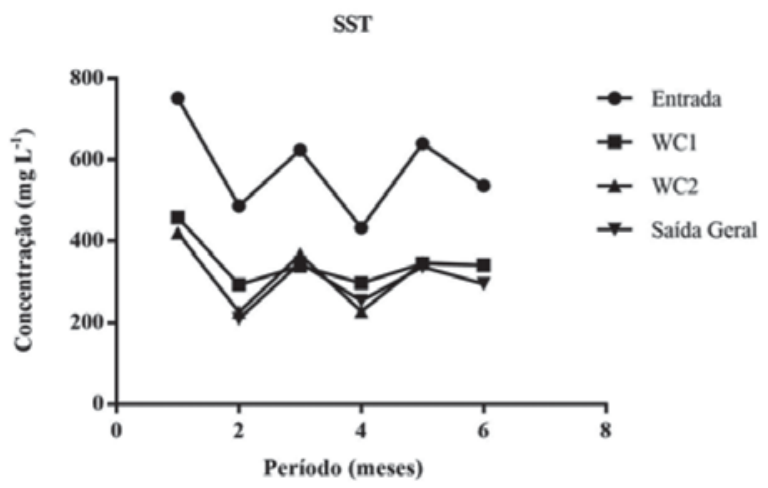

SSF
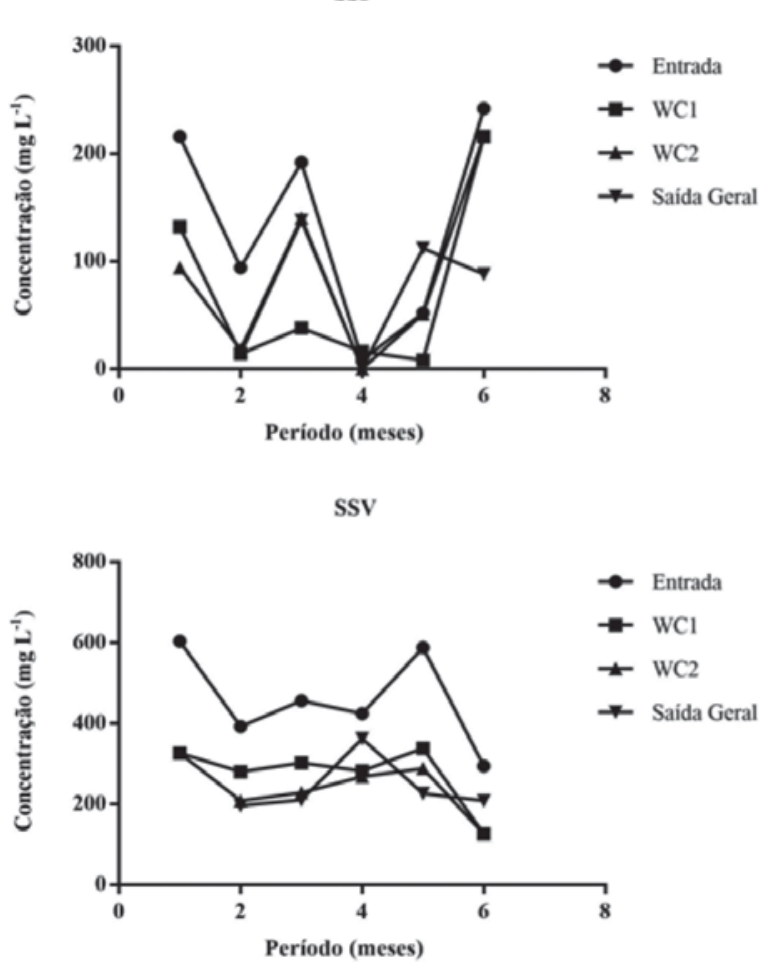

Figura 4 - Série temporal das concentrações efluentes de SST, SSV e SSF.

Sperling, 2013; Von Sperling, 2014).

Ainda tratando dos riscos do aporte excessivo de sólidos no leito filtrante, ou seja, a obstrução dos poros, tendo, agora como base a taxa de carregamento de sólidos recomendável para WHFSS, a USEPA(2000) e o German Guideline ATV-AG62, citados por Wojciechowska et al. (2010), sugerem concentração máxima no afluente de $100 \mathrm{mg} \mathrm{L}^{-1}$ de SST. 
Com um aporte médio de $578 \mathrm{mg} \mathrm{L}^{-1}$ de SST nas unidades de wetlands construídos, aproximadamente um valor seis vezes superior ao recomendado, pode, em longo prazo, diminuir a eficiência do sistema. Essa retenção de sólidos nos sistemas de wetlands construídos ocorre principalmente através do processo de coagem das partículas no meio poroso que atua como um verdadeiro meio filtrante (Kadlec \& Wallace, 2009; Ansari et al., 2016). Com uma eficiência média de 41, 44 e 50\% para as unidades WC1, WC2 e WC geral, respectivamente na retenção dos sólidos suspensos totais, os wetlands construídos foram alvo do fenômeno natural da colmatação, o qual teve suas primeiras aparições no terceiro mês do monitoramento (outubro), ou no oitavo mês de seu funcionamento. Este fenômeno foi identificado com o aparecimento de escoamento superficial nas unidades, no qual deveria ser subsuperficial.

$\mathrm{O}$ alto aporte de SST (578 $\mathrm{mg} \mathrm{L}^{-1}$ ) diminuiu consideravelmente a porosidade do material filtrante presente nas unidades de WC deste estudo, em que foi registrado os primeiros indícios de escoamento superficial no mês de outubro em uma das quatro unidades de WC. Já nos meses seguintes também apresentaram escoamento superficial nas demais unidades e, com aproximadamente um ano de operação (janeiro 2017) as unidades de WC não suportaram a alta sobrecarga dos sólidos presente no esgoto doméstico, sendo necessário a interrupção de duas unidades para fazer a primeira intervenção. A intervenção foi executada, substituindo o material filtrante, areia por pedrisco, a fim de aumentar a condutividade hidráulica na última etapa do leito filtrante e consequentemente uma maior permeabilidade do meio filtrante.

\section{Nitrogênio total}

As concentrações analisadas quanto à nitrogênio total ( $\left.\mathrm{N}_{\text {Total }}\right)$, estão apresentados pela série temporal na Figura 5.

Em relação ao nitrogênio total, as unidades WC apresentaram comportamento inconstante ao longo do período do monitoramento. O efluente, após o filtro anaeróbio, apresentava concentrações médias de $\mathrm{N}_{\text {Total }}$ de 126,5 $\mathrm{mg} \mathrm{L}^{-1}$. Após o tratamento via WC, o efluente geral da ETE apresentou concentrações médias de 86,2 $\mathrm{mg} \mathrm{L}{ }^{-1}$, conferindo uma eficiência média de remoção de $31,8 \%$.

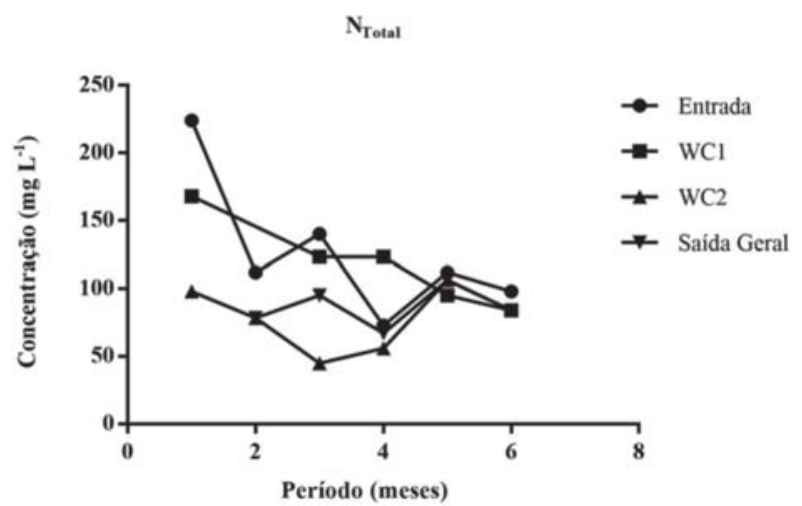

Figura 5 - Série temporal da concentração efluente de $\mathrm{N}_{\text {Total }}$ para os wetlands construídos.

Durante o período do monitoramento, de aproximadamente seis meses, foram registradas remoções médias de 6,1, 38,4 e 31,8\% de $\mathrm{N}_{\text {Total }}$ para as unidades WC1, WC2 e WC geral, respectivamente. Avaliando as concentrações dos efluentes das unidades de wetlands construídos a unidade WC2 obteve a maior eficiência de remoção. As remoções temporais das concentrações efluentes de $\mathrm{N}_{\text {Total }}$ revelam comportamento semelhantes: remoção muito elevada no início do monitoramento do sistema, demostrando a capacidade do sistema em amortecer as cargas afluentes do filtro anaeróbio, seguida, entretanto, de ciclos de queda de desempenho. De modo geral, as unidades de WC nos últimos três meses registraram as menores eficiências, inclusive, episódios de concentrações efluentes de $\mathrm{N}_{\text {Total }}$ superiores às dos afluentes, com destaque ao WC1, apresentando uma eficiência negativa (-69\%) no quarto mês de monitoramento, esse episódio contribuiu para que essa unidade apresentasse o menor desempenho perante às demais. (Figura 6).

Segundo De Paoli (2010) o escoamento superficial indica o princípio da colmatação do sistema e que este fenômeno natural dos wetlands construídos de fluxo subsuperficial diminui o tempo do efluente dentro da unidade e consequentemente, a eficiência na remoção de matéria orgânica e nutrientes, principalmente o nitrogênio.

Os valores de eficiência de remoção de nitrogênio apresentados na literatura são bastante amplos, e variam de acordo com o tempo de operação dos sistemas e do tempo de detenção hidráulico utilizado em cada WC. Sousa et al. (2004), avaliando um wetland de mesma 

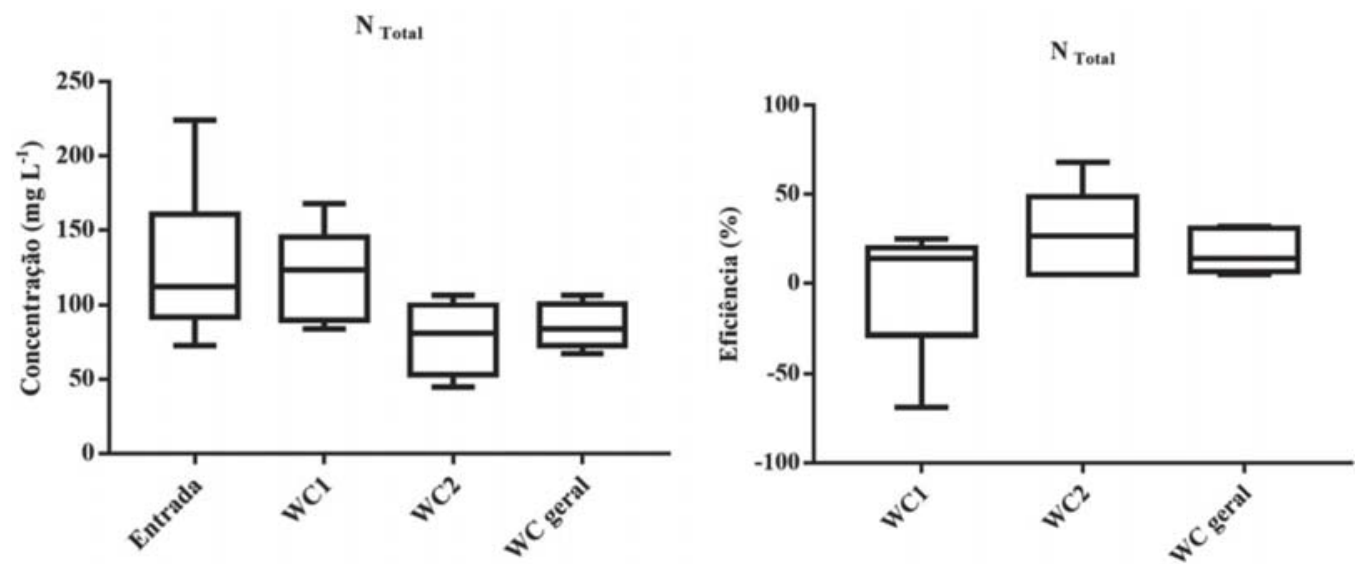

Figura 6 - Gráfico tipo boxplot, destacando as concentrações efluentes e das eficiências de remoção do $\mathrm{N}_{\text {Total }}$ no filtro anaeróbio e nos wetlands construídos.

característica desta pesquisa, encontraram grandes oscilações temporais, com queda de desempenho ao longo do período de operação do sistema e obtiveram eficiência média de $60 \%$ de remoção de $\mathrm{N}_{\text {Total }}$ para o primeiro ano de operação. Outros trabalhos também reportam tendência de remoção inicial elevada seguida de queda (Vymazal, 1996, 2004). De Paoli (2010), ao avaliar um WC horizontal cultivado com Taboa, no tratamento de esgoto doméstico, também observou baixa eficiência na remoção de $\mathrm{N}_{\text {Total }}(13 \%)$ no primeiro ano de monitoramento.

As cargas de nitrogênio aplicadas aos WC e o tipo de escoamento apresentado pelos WC são os principais fatores de influência na eficiência de remoção desse nutriente, que, de acordo com Vymazal (2007), essas eficiências variam entre 40 e 50\%. Ainda segundo esse autor, um WC de único estágio não pode alcançar maiores eficiências na remoção de nitrogênio em razão da sua dificuldade em proporcionar condições anaeróbias e aeróbias em um mesmo ambiente.

Os principais mecanismos responsáveis pela remoção de nitrogênio em WC são: nitrificaçãodesnitrificação, sedimentação de nitrogênio orgânico, assimilação por plantas, assimilação por microrganismos e a volatilização da amônia (Sezerino, 2006; Vymazal \& Kroepfelová, 2008). Entretanto, permanecem incertezas sobre os mecanismos predominantes de remoção, cujas contribuições, muito provavelmente, variam segundo a configuração de cada sistema e as condições climáticas.

\section{Fósforo total}

As concentrações analisadas quanto ao fósforo total $\left(\mathrm{P}_{\text {Total }}\right)$, estão apresentados pela série temporal na Figura 7.

Em relação ao fósforo total, as unidades WC apresentaram comportamento inconstante ao longo do período de monitoramento. O efluente, após o filtro anaeróbio, apresentava concentrações médias de $\mathrm{P}_{\text {Total }}$ de 3,1 $\mathrm{mg} \mathrm{L}^{-1}$, após o tratamento via WC, o efluente dos WC1, WC2 e WC geral apresentaram concentrações médias de 2,81 $\mathrm{mg} \mathrm{L}^{-1}, 2,12 \mathrm{mg} \mathrm{L}^{-1}$ e 2,8 $\mathrm{mg} \mathrm{L}^{-1}$, respectivamente. A remoção média de $\mathrm{P}_{\text {Total }}$ nos wetlands foram de 13,5, 35,2 e 22,4\% para WC1, WC2 e WC geral, respectivamente.

A resolução CONAMAn ${ }^{\circ}$ 430/2011 não faz referência aos limites de $\mathrm{P}_{\text {Total }}$ quanto ao descarte de efluente doméstico em corpo hídrico, apenas a Lei estadual do Estado de Santa Catarina n ${ }^{\circ}$ 14675/2009 (FATMA) que limita a concentração de $\mathrm{P}_{\text {Total }}$ no efluente em $4 \mathrm{mg} \mathrm{L}^{-}$ ${ }^{1}$. Todas as concentrações dos efluentes dos WC foram inferiores ao valor padrão determinado pela legislação vigente, exceto no quinto mês de monitoramento em que a concentração de $\mathrm{P}_{\text {Total }}$ foi superior a $4 \mathrm{mg} \mathrm{L}^{-1}$ nas unidades de WC1 e WC geral.

No início do monitoramento foi registrado eficiência de remoção de $\mathrm{P}_{\text {Total }}$ muito elevada, porém seguiramse períodos de queda e ganho de desempenho do sistema. Trabalhos semelhantes como de Sousa et al. (2004), De Paoli (2010) e Secchi et al. (2016) reportaram 80, 
33 e 38,4\%, respectivamente, para remoção média de $\mathrm{P}_{\text {Total }}$ em WC horizontais no tratamento de esgoto doméstico.

Com relação ao fósforo, os principais mecanismos de remoção em WC são: armazenamento na biomassa vegetal e microbiológica, precipitação de compostos insolúveis e adsorção ao substrato (Vymazal \& Kroepfelová, 2008; Von Sperling, 2015). Vymazal (2011) afirma que o principal mecanismo de remoção de P é via precipitação de compostos e adsorção ao substrato com um potencial de remoção entre 40 e $60 \%$, podendo ser maior quando o substrato possuir elevada capacidade de sorção. Atribui-se, portanto, ao potencial do meio filtrante como principal responsável pela remoção do fósforo presente no efluente.

De maneira geral, os meses iniciais apresentaram melhores resultados quanto a remoção de $\mathrm{P}_{\text {Total }}$ nos WC. Essa ocorrência pode ser em função da assimilação das plantas pelo alastramento da cultura da Taboa sob a área do leito, bem como do desenvolvimento da biomassa microbiana no meio filtrante. Cabe destacar que para a remoção de fósforo, assim como outros parâmetros (nitrogênio e coliformes), o tempo de contato do efluente com o meio possui papel importante para a remoção no interior dos wetlands construídos, seja pela absorção das macrófitas ou por meio das reações químicas que ocorrem entre o elemento e o meio filtrante. Porém, a partir do quarto mês do monitoramento há o decaimento da eficiência de depuração deste elemento nas unidades de wetlands construídos. Essa menor

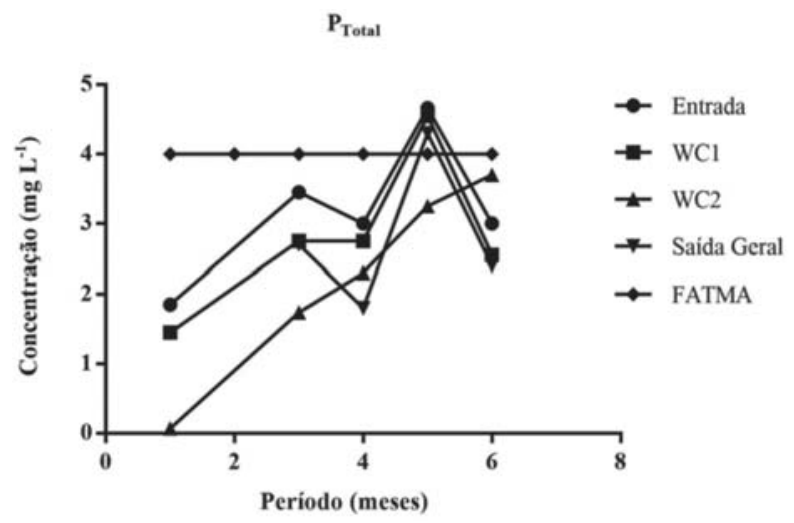

Figura 7 - Série temporal da concentração efluente de $\mathrm{P}_{\text {Total }}$ para os wetlands construídos $\mathrm{e}$ comparação com a Lei do estado de SC (FATMA). eficiência, principalmente na unidade WC1 (13,5\%), pode estar relacionada com o menor tempo de detenção que o efluente possa ter ficado dentro das unidades, devido à formação de caminhos preferenciais e do escoamento superficial, este ocasionado pelo fenômeno natural da colmatação (De Paoli, 2010; De Paoli \& Von Sperling, 2013; Von Sperling, 2015).

\section{Coliformes totais}

A Figura 8 apresenta a série temporal das concentrações efluentes de coliformes totais (CT).

As remoções médias de CT nos wetlands construídos foram de 73,1, 88,5 e 69,7\% para WC1, WC2 e WC geral, respectivamente. Ou seja, as unidades de WC apresentaram remoções de 1 a 2 unidades logarítmicas. Os principais mecanismos responsáveis pela remoção dos CT nos WC são a filtração, sedimentação, predação por outros organismos e, morte natural (Ansari et al., 2016). Além desses mecanismos comuns, as plantas favorecem melhores condições para a remoção dos CT, segundo De Paoli (2010) as unidades com plantas podem apresentar melhor desempenho em função da liberação de biocidas e oxigênio pelas raízes e de conter maior diversidade de microrganismos predadores.

As eficiências de remoção de CT alcançados nesta pesquisa foram condizentes com os resultados descritos em USEPA (2000) e Ansari et al. (2016), onde se afirmam que a remoção em sistemas de WC é em torno de duas unidades logarítmicas de coliformes totais.

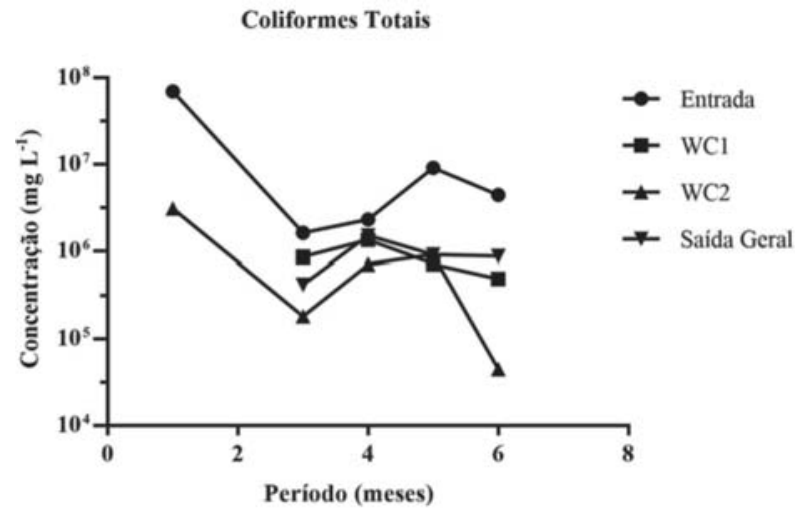

Figura 8 - Série temporal da concentração efluente de CT para os wetlands construídos. 


\section{CONCLUSÃO}

A ETE Aparecida está de acordo com as legislações vigentes quanto ao descarte de efluente doméstico a corpo hídrico (Resolução CONAMA n 430/2011 e Lei estadual $n^{\circ} 14675 / 2009$ (FATMA)) em todos os parâmetros avaliados, exceto DBO e $\mathrm{P}_{\text {Total }}$. Estes não atenderam aos padrões estabelecidos pela FATMA nos dois últimos meses, mas estavam abaixo dos limites estabelecidos pelo CONAMA.

Apesar da precoce manutenção corretiva e preventiva devido à ocorrência de colmatação, a utilização de wetlands construídos de escoamento horizontal de fluxo subsuperficial é uma alternativa para o tratamento de esgoto doméstico para a região de Campos Novos, SC.

\section{LITERATURACITADA}

ANSARI, A.A.; GILL, R.; GILL, S.S. et al.

Phytoremediation. Management of Environmental Contaminants, v.4. Suíça: Springer, 2016.

AMERICAN PUBLIC HEALTH ASSOCIATION. Standard methods for the examination of water and wastewater. 20 ed. Washington: APHA, 1998. 937p.

BRASIL. Resolução $\mathbf{n}^{\circ} \mathbf{4 3 0}$, de 13 de maio de 2011. Dispõe sobre as condições de lançamento de efluentes, complementa e altera a Resolução n 357, de 17 de março de 2005, do Conselho Nacional do Meio Ambiente CONAMA. 9p. 75. Disponível em: < http:// www.mma.gov.br/port/conama/ legiabre.cfm?codlegi $=646$. $>$ (acessado em 29 de janeiro de 2018).

\section{Lei $n^{\circ} 11.445$ de 5 de janeiro}

de 2007. Estabelece diretrizes nacionais para o saneamento básico; altera as Leis noㅗ 6.766, de 19 de dezembro de 1979, 8.036, de 11 de maio de 1990, 8.666, de 21 de junho de 1993, 8.987, de 13 de fevereiro de 1995; revoga a Lei no 6.528, de 11 de maio de 1978; e dá outras providências. Brasília, DF, jan 2007. Disponível em: < http:// www.planalto.gov.br/ccivil_03/_ato2007-2010/ 2007/lei/l11445.htm> (acessado em 21 de março de 2018).
CAMPOS NOVOS (Município). Lei no 3.941, de 26 de setembro de 2013. Estabelece normas de saneamento básico para a implantação de loteamentos no município de Campos Novos e dá outras providências. Campos Novos, SC. Disponível em: <http://edicao.dom.sc.gov.br/pdfjs/web/ viewer.html?file=http\%3A\%2F\%2Fedicao. dom.sc.gov.br\%2F0.976651001380575059_edicao_ dom_1336_13.pdf\#page=42> (acessado em 29 de janeiro de 2018).

COLARES, C.J.G.; SANDRI, D. Eficiência de tratamento de esgoto com tanques sépticos seguidos de leitos cultivados com diferente meio suporte. Revista Ambiente \& Água, v.8, n.1, p.172-185, 2013.

DE PAOLI, A.C. Análise de desempenho e comportamento de wetlands horizontais de fluxo subsuperficial baseado em modelos hidráulicos e cinéticos. Dissertação (Mestrado em Saneamento, Meio Ambiente e Recursos Hídricos). Belo Horizonte, MG: UFMG, 2010.

DE PAOLI, A.C.; VON SPERLING, M. Avaliação das condições hidrodinâmicas de wetlands de escoamento horizontal subsuperficial (unidades plantada e não plantada). Revista Eletrônica de Gestão e Tecnologias Ambientais, v.1, n.2, p.213-222, 2013.

IBGE - Instituto Brasileiro de Geografia e Estatística. Estimativas da população residente no Brasil e unidades da federação com data de referência em $1^{\circ}$ de julho de 2017. 2017. Disponível em: ftp://ftp.ibge.gov.br/Estimativas_de_Populacao/ Estimativas_2017/

estimativa_TCU_2017_20180112.pdf > (acessado em 23 de janeiro de 2018).

KADLEC, R.H.; WALLACE, S.R. Treatment Wetlands, 2nd edition. Boca Raton, Florida: CRC Press, 2009.

KIVAISI, A.K. The potential for constructed wetlands for wastewater treatment and reuse in developing countries: a review. Ecological Engineering, v.16, p.545-560, 2001.

KONRAD, O.; TONETTO, J.F.; SIQUEIRA, L.M. et al. Avaliação da eficiência de wetland construído para tratamento de efluente proveniente de vinícola. Revista Brasileira de Viticultura e Enologia, n.7, p.10-17, 2015. 
MACÊDO, J.A.B. Métodos laboratoriais de análises fisico-químicas e microbiológicas. $4^{\mathrm{a}}$ Edição. Belo Horizonte: CRQ-MG, 2013.

PHILIPPI, L.S.; SEZERINO, P.H. Aplicação de sistemas tipo wetlands no tratamento de águas residuárias: utilização de filtros plantados com macrófitas. Florianópolis: Ed. do Autor, 2004. p.144.

SANTA CATARINA (Estado). Lei $\mathbf{n}^{\circ}$ 14.675, de 13 de abril de 2009. Institui o Código Estadual do Meio Ambiente e estabelece outras previdências. Santa Catarina, 2009.

SECCHI, F.J.; KONRAD, O.; TONETTO, J. Avaliação do balanço hídrico e da eficiência de um alagado construído como alternativa de tratamento para efluente doméstico. Ciência e Natura, v.38, n.1, p.453-461, 2016.

SEZERINO, P.H. Potencialidade dos filtros plantados com macrófitas (constructed wetlands) no pós-tratamento de lagoas de estabilização sob condições de clima subtropical. Tese (Doutorado em Engenharia Ambiental). Florianópolis, SC: UFSC, 2006.

SNIS, Sistema Nacional de Informações sobre Saneamento. Diagnóstico dos Serviços de Água e Esgoto. MCidades. 2015. Disponível em: <http:// www.snis.gov.br/diagnostico-agua-e-esgotos/ diagnostico-ae-2014> (acessado em 25 de maio de 2017).

SOUZA, A.L. Estudo experimental e numérico do processo de remoção de poluentes nos leitos cultivados. Dissertação (Mestrado em Engenharia Agrícola). Campinas, SP: UNICAMP, 2003.

Disponível em: <http://repositorio.unicamp.br/ bitstream/REPOSIP/257561/1/

Souza_AndersonLuizde_M.pdf $>$ (acessado em: 19 de janeiro de 2018).

SOUSA. J.T.; HAANDEL, A.V.; LIMA, E.P.C. et al. Utilização de wetland construído no póstratamento de esgotos domésticos pré-tratados em reator UASB. Engenharia Sanitária e Ambiental, v.9, n.4, p.285-290, 2004.
U.S. EPA. Constructed wetlands treatment of municipal wastewater. Manual. EPA 625/R-99/ 010, U.S. Environmental Protection Agency, Cincinnati, Ohio, 2000.

VON SPERLING, M. Introdução à qualidade das águas e ao tratamento de esgotos. Editora UFMG, 2014.

VON SPERLING, M. Comparison of simple, small, full-scale sewage treatment systems in Brazil: UASB-maturation ponds-coarse filter; UASB horizontal subsurface-flow wetland; vertical-flow wetland (first stage of French system). Water Science Technology, v.71, n.3, p.329, 2015.

VYMAZAL, J. Constructed wetlands for wastewater treatment in the Czech Republic the first 5 years experience. Water Science and Technology, v.34, n.11, p.159-164, 1996.

VYMAZAL, J. Removal of phosphorus in constructed wetlands with horizontal subsurface flow in the Czech Republic. Water, Air, \& Soil Pollution: Focus, v.4, n.2-3, p.657670, 2004.

VYMAZAL, J. Removal of nutrients in various types of constructed wetlands. Science of the Total Environment, v.380, p.48-65, 2007.

VYMAZAL, J.; KROPFELOVÁ, L. Wastewater treatment in constructed wetlands with horizontal sub-surface flow. República Tcheca: Springer, 2008.

VYMAZAL, J. Long-term performance of constructed wetlands with horizontal subsurface flow: Tem case studies from the Czech Republic. Ecological Engineering, v.37, p.54-63, 2011.

WOJCIECHOWSKA, E.; GAJEWSKA, M.; OBARSKA-PEMPKOWIAK, H. Treatment of Landfill Leachate by Constructed Wetlands:

Three Case Studies. Polish Journal of

Environmental Studies, v.19, n.3, p.643-650, 2010.

Recebido para publicação em 30/1/2018 e aprovado em 29/3/2018. 